\title{
Intracortical Dynamics Underlying Repetitive Stimulation Predicts Changes in Network Connectivity
}

\author{
ำYuhao Huang, ${ }^{1,2}$ Boglárka Hajnal, ${ }^{3,4}$ László Entz, ${ }^{3}$ @Dániel Fabó, ${ }^{3,4}$ Jose L. Herrero, ${ }^{5,6}$ @Ashesh D. Mehta, ${ }^{5,6}$ \\ and $\oplus^{-C o r e y ~ J . ~ K e l l e r ~}{ }^{1,2,5}$ \\ ${ }^{1}$ Department of Psychiatry and Behavioral Sciences, Stanford University Medical Center, Stanford, California 94305, ${ }^{2}$ Veterans Affairs Palo Alto Healthcare \\ System, and the Sierra Pacific Mental Illness, Research, Education, and Clinical Center, Palo Alto, California 94394, ${ }^{3}$ Department of Functional \\ Neurosurgery and Department of Epilepsy, National Institute of Clinical Neuroscience, Budapest, Hungary 1145, ${ }^{4}$ Szentágothai János School of PhD studies, \\ Semmelweis University, Budapest, Hungary 1085, ${ }^{5}$ Department of Neurosurgery, Hofstra Northwell School of Medicine, and ${ }^{6}$ Feinstein Institute for Medical \\ Research, Manhasset, New York 11030
}

Targeted stimulation can be used to modulate the activity of brain networks. Previously we demonstrated that direct electrical stimulation produces predictable poststimulation changes in brain excitability. However, understanding the neural dynamics during stimulation and its relationship to poststimulation effects is limited but critical for treatment optimization. Here, we applied $10 \mathrm{~Hz}$ direct electrical stimulation across several cortical regions in 14 human subjects ( 6 males) implanted with intracranial electrodes for seizure monitoring. The stimulation train was characterized by a consistent increase in high gamma $(70-170 \mathrm{~Hz})$ power. Immediately post-train, low-frequency $(1-8 \mathrm{~Hz})$ power increased, resulting in an evoked response that was highly correlated with the neural response during stimulation. Using two measures of network connectivity, corticocortical evoked potentials (indexing effective connectivity), and theta coherence (indexing functional connectivity), we found a stronger response to stimulation in regions that were highly connected to the stimulation site. In these regions, repeated cycles of stimulation trains and rest progressively altered the stimulation response. Finally, after just $2 \mathrm{~min}(\sim 10 \%)$ of repetitive stimulation, we were able to predict poststimulation connectivity changes with high discriminability. Together, this work reveals a relationship between stimulation dynamics and poststimulation connectivity changes in humans. Thus, measuring neural activity during stimulation can inform future plasticity-inducing protocols.

Key words: cortico-cortical evoked potentials; electrical stimulation; electrocorticography; neuromodulation; plasticity; repetitive stimulation

Significance Statement

Brain stimulation tools have the potential to revolutionize the treatment of neuropsychiatric disorders. Despite the widespread use of brain stimulation techniques such as transcranial magnetic stimulation, the therapeutic efficacy of these technologies remains suboptimal. This is in part because of a lack of understanding of the dynamic neural changes that occur during stimulation. In this study, we provide the first detailed characterization of neural activity during plasticity induction through intracranial electrode stimulation and recording in 14 medication-resistant epilepsy patients. These results fill a missing gap in our understanding of stimulation-induced plasticity in humans. In the longer-term, these data will also guide our translational efforts toward non-invasive, personalized, closed-loop neuromodulation therapy for neurological and psychiatric disorders in humans.

\section{Introduction}

Brain stimulation treatments including repetitive transcranial magnetic stimulation (rTMS) have recently emerged as clinically-

Received March 7, 2019; revised May 12, 2019; accepted May 24, 2019.

Author contributions: C.J.K. designed research; Y.H., B.H., L.E., D.F., J.L.H., A.D.M., and C.J.K. performed research; Y.H. and C.J.K. analyzed data; Y.H. and C.J.K. wrote the paper.

This work was supported by the National Institute of Neurological Disorders and Stroke (F31NS080357-01 and T32-GM007288), Stanford Society of Physician Scholars Collaborative Research Fellowship, and Alpha Omega Alpha Postgraduate Research Award to C.J.K., and by the Hungarian National Research, Development, and Innovation Office (2017-1.2.1-NKP-2017-00002) to D.F. We thank Maria Fini and Victor Du for help with data collection, Pierre Megevand and Erin Yeagle for help with technical considerations of the experimental design, and Wei Wu for effective alternatives to medications for neuropsychiatric disorders. Although rTMS is FDA-approved for certain disorders (major depression, obsessive-compulsive disorder) and there are

comments on the paper. All authors discussed the data, analysis, and methods and contributed to the paper. The authors are enormously indebted to the patients who participated in this study, as well as the nursing and physician staff at North Shore University Hospital (Manhassat, NY) and the National Institute of Clinical Neurosciences (Budapest, Hungary).

The authors declare no competing financial interests.

Correspondence should be addressed to Corey J. Keller at ckeller1@stanford.edu.

https://doi.org/10.1523/JNEUROSCI.0535-19.2019

Copyright $\odot 2019$ the authors 
multiple ongoing clinical trials for other disorders (posttraumatic stress disorder, substance use, epilepsy), the mechanism of how rTMS induces neural plasticity remains poorly understood. Studying brain dynamics during stimulation could provide a crucial set of principles to optimize rTMS and other neuromodulation treatments (e.g., deep-brain stimulation and vagus nerve stimulation).

The dynamics of neuronal plasticity are typically separated into the induction phase (changes during stimulation) and the maintenance phase (changes lasting minutes to hours after stimulation). The maintenance phase is characterized by a large change in synaptic response that persists for hours and involves modifications in gene expression, protein synthesis and synaptic function (Bliss and Lomo, 1973; Huang et al., 1992; Mulkey and Malenka, 1992; Bliss and Collingridge, 1993, Liu et al., 1999; Miyamoto, 2006). The induction period has been less well characterized, but in animal models changes observed during stimulation (induction phase) appear to relate to poststimulation maintenance effects. During tetanic stimulation in rat hippocampal slices, synaptic responses dynamically changed as more pulses were applied (Wójtowicz and Mozrzymas, 2014), and the magnitude of maintenance effects correlated with changes during stimulation. In nonhuman primates, $>5 \mathrm{~Hz}$ optogenetic stimulation to the sensorimotor cortices progressively increased functional connectivity during stimulation and predicted changes in poststimulation connectivity (Yazdan-Shahmorad et al., 2018). Furthermore, $10 \mathrm{~Hz}$ magnetic stimulation (rTMS) to cat visual cortices elicited a pulse-wise increase in neural activity, resulting in significantly increased poststimulation evoked and spontaneous activity (Kozyrev et al., 2014). These findings in animal models suggest discrete neural changes occur during induction that relate to poststimulation changes.

Few studies have examined the induction phase in humans. Two studies have recorded scalp electroencephalogram (EEG) while applying noninvasive rTMS and reported changes in the evoked potential during stimulation (Hamidi et al., 2010; Veniero et al., 2010). However, because of the short latency of these evoked potentials and the possibility of residual stimulationrelated artifacts, the interpretation of these findings is limited (Rosanova et al., 2012; Wu et al., 2018). In contrast, invasive recordings provide high spatiotemporal resolution with temporally defined artifact, allowing precise measurement of neural activity after each pulse. Recent studies using direct electrical stimulation coupled with invasive EEG demonstrated evidence of entrainment to the stimulation frequency (Amengual et al., 2017), decrease in low-frequency power during high-frequency stimulation ( $100 \mathrm{~Hz}$; Rao et al., 2018), and increased theta activity directly after a stimulation train (Solomon et al., 2018). We recently demonstrated that repeated $10 \mathrm{~Hz}$ electrical stimulation resulted in poststimulation excitability changes in regions functionally connected to the stimulation site (Keller et al., 2018). Furthermore, tracking the first pulse across stimulation trains was useful in predicting these poststimulation changes, suggesting a potential link between the induction period and maintenance effects. Overall, human studies have begun to explore the complex dynamics of the induction phase of plasticity, but a detailed characterization is still lacking.

In this investigation, we sought to better understand (1) the neural response to a stimulation train and (2) how brain activity during stimulation relates to poststimulation connectivity changes. We hypothesized that a stimulation train would increase neural responses in regions functionally connected to the stimu- lation site, and the strength of response during stimulation could be used to predict poststimulation connectivity changes. We found that prestimulation connectivity predicted the stimulation response, which involves an increase in high-frequency (70-170 $\mathrm{Hz}$ ) power, followed by a low-frequency $(1-8 \mathrm{~Hz})$ power evoked response. In regions highly connected to the stimulation site, progressive change of the stimulation response was observed during the course of stimulation. Using features from the stimulation period, we were able to predict poststimulation connectivity changes with high discriminability. This work demonstrates the feasibility of measuring neural activity during repetitive stimulation and serves to inform stimulation-based therapies for neuropsychiatric disorders.

\section{Materials and Methods}

Participants. Fourteen patients (6 males) with medically-intractable epilepsy underwent surgical implantation of intracranial electrodes for seizure localization. Patient characteristics are described in Table 1. Patients were enrolled at two hospitals: North Shore University Hospital (Manhasset, New York) and National Institute of Clinical Neurosciences (Budapest, Hungary). Eight patients from the current study was included in a prior study on poststimulation effects of repetitive brain stimulation (Keller et al., 2018). No analyses described in the current study were performed in the prior study. All patients provided informed consent as monitored by the local Institutional Review Board and in accordance with the ethical standards of the Declaration of Helsinki. The decision to implant, the electrode targets, and the duration of implantation were made entirely on clinical grounds without reference to this investigation. Patients were informed that participation in this study would not alter their clinical treatment, and that they could withdraw at any time without jeopardizing their clinical care.

Electrode registration. Our electrode registration method has been described in detail previously (Dykstra et al., 2012; Groppe et al., 2017). Briefly, to localize each electrode anatomically, subdural electrodes were identified on the post-implantation CT with BioImage Suite, (Duncan et al., 2004) and were coregistered first with the post-implantation structural MRI and subsequently with the pre-implantation MRI to account for possible brain shift caused by electrode implantation and surgery (Mehta and Klein, 2010). Following automated coregistration, electrodes were snapped to the closest point on the reconstructed pial surface (Dale et al., 1999) of the pre-implantation MRI in MATLAB (Dykstra et al., 2012). Intraoperative photographs were previously used to corroborate this registration method based on the identification of major anatomical features. Automated cortical parcellations were used to localize electrodes to anatomical regions (Fischl et al., 2004).

Electrophysiological recordings. Invasive electrocorticographic (ECoG) recording from implanted intracranial subdural grids, strips, and/or depth electrodes were sampled at 512 or $2048 \mathrm{~Hz}$ depending on clinical parameters at the participating hospital (U.S. and Hungary, respectively). Data preprocessing and analysis was performed using the FieldTrip toolbox (Oostenveld et al., 2011). Line noise (60 and $50 \mathrm{~Hz}$ for recordings in the U.S. and Hungary, respectively) was removed using a notch filter. Direct electrical stimulation induced stereotyped stimulation artifacts that were $\sim 30 \mathrm{~ms}$ in duration. We applied a fourth-order bandpass filter (Butterworth, two-pass) in the 100-150 Hz frequency range, which sharply localizes the stimulation artifacts as these artifacts comprise primarily high-frequency power $(>40 \mathrm{~Hz}$; Fig. $1 D)$. Stimulation artifacts were subsequently detected by applying a value threshold. This value threshold was chosen per subject to detect all stimulation artifacts within the stimulation train. We replaced the stimulation artifact with stationary ECoG time series that represented similar amplitude and spectral profile as the background signal. This procedure was detailed previously (Crowther et al., 2019) and is preferred over simple spline interpolation given short intervals between pulses and the potential to introduce large spectral changes. To do this, we extracted ECoG signal with equal length as the stimulation artifact ( $\sim 30 \mathrm{~ms}, 15$ or 61 samples depending on the sampling frequency) immediately preceding 
Table 1. Participant characteristics, implant type, and stimulation sites

\begin{tabular}{|c|c|c|c|c|c|c|c|c|}
\hline$\overline{I D}$ & Age & Sex & Handedness & Medications & Seizure zone & Implant type & Stimulation location & $\mathrm{MNI}$ \\
\hline S1 & 21 & M & R & $\begin{array}{l}\text { Levetiracetam } \\
\text { Lacosamide }\end{array}$ & R mesial temporal & Grid/strips & R precentral gyrus & $60,-12,39$ \\
\hline S2 & 57 & $\mathrm{~F}$ & L & $\begin{array}{l}\text { Levetiracetam } \\
\text { Carbamazepine }\end{array}$ & $\mathrm{R}$ mesial temporal & sEEG & L precentral gyrus & $-58,-6,39$ \\
\hline S3 & 31 & $\mathrm{~F}$ & $\mathrm{R}$ & $\begin{array}{l}\text { Levetiracetam } \\
\text { Carbamazepine } \\
\text { Clonazepam }\end{array}$ & RSTG/mesial temporal & sEEG & R precentral gyrus & $57,-13,37$ \\
\hline S4 & 43 & $\mathrm{~F}$ & $\mathrm{R}$ & $\begin{array}{l}\text { Carbamazepine } \\
\text { Zonisamide }\end{array}$ & R posterior temporal & Grid/strips & L middle frontal gyrus & $-44,35,29$ \\
\hline S5 & 30 & $\mathrm{~F}$ & $\mathrm{R}$ & Lamotrigine & L mesial temporal & Grid/strips & L middle temporal gyrus & $-35,27,-29$ \\
\hline S6 & 32 & M & $\mathrm{L}$ & $\begin{array}{l}\text { Carbamazepine } \\
\text { Clobazam } \\
\text { Topiramate }\end{array}$ & $\mathrm{R}$ middle frontal gyrus & Grid/strips & R precentral gyrus & $65,-1,19$ \\
\hline S7 & 20 & M & $\mathrm{R}$ & $\begin{array}{l}\text { Levetiracetam } \\
\text { Carbamazepine } \\
\text { Lamotrigine }\end{array}$ & R frontal cortex & Grid/strips & R precentral gyrus & $65,-5,23$ \\
\hline S8 & 44 & $\mathrm{~F}$ & $\mathrm{R}$ & $\begin{array}{l}\text { Carbamazepine } \\
\text { Valproate } \\
\text { Lamotrigine }\end{array}$ & R premotor cortex & sEEG & R middle frontal gyrus & $55,28,17$ \\
\hline S9 & 28 & $\mathrm{~F}$ & $\mathrm{R}$ & $\begin{array}{l}\text { Levetiracetam } \\
\text { Carbamazepine } \\
\text { Lamotrigine }\end{array}$ & R hippocampus & sEEG & R temporo-parieto-occipital junction & $51,-43,21$ \\
\hline S10 & 28 & M & R & $\begin{array}{l}\text { Levetiracetam } \\
\text { Lamotrigine } \\
\text { Clonazepam }\end{array}$ & L middle frontal gyrus & sEEG & R inferior frontal gyrus & $19,37,-21$ \\
\hline S11 & 36 & M & $\mathrm{R}$ & $\begin{array}{l}\text { Levetiracetam } \\
\text { Lamotrigine }\end{array}$ & L temporo-polar-basal & sEEG & L mesial temporal & $-23,9,-32$ \\
\hline S12 & 50 & $\mathrm{~F}$ & $\mathrm{R}$ & $\begin{array}{l}\text { Levetiracetam } \\
\text { Topiramate }\end{array}$ & R OFC/amygdala & sEEG & $\mathrm{R}$ cingulate gyrus & $6,37,13$ \\
\hline S13 & 48 & $\mathrm{~F}$ & $\mathrm{R}$ & $\begin{array}{l}\text { Levetiracetam } \\
\text { Clobazam } \\
\text { Topiramate } \\
\text { Phenytoin } \\
\text { Primidone }\end{array}$ & R mesial temporal & sEEG & R middle frontal gyrus & $55,35,13$ \\
\hline S14 & 46 & M & $\mathrm{R}$ & $\begin{array}{l}\text { Levetiracetam } \\
\text { Valproate } \\
\text { Topiramate } \\
\text { Lacosamide }\end{array}$ & R posterior temporal & sEEG & L inferior frontal gyrus & $-51,13,4$ \\
\hline
\end{tabular}

and following the artifact (Fig. 1C). We reversed the ECoG signal and applied a tapering matrix $(1: 1 / n: 0$ for the preceding data, $0: 1 / n: 1$ for the following data, where $n$ is the number of samples contained in the artifact). The two ECoG signals were added together and subsequently used to replace the artifact period. The effectiveness of this artifact removal process is shown in Figure 1. Following artifact rejection, we applied a bipolar montage to depth electrodes and a common average reference montage to grid electrodes (Stolk et al., 2018).

Repetitive stimulation paradigm. To examine cortical dynamics during and after stimulation, we applied focal $10 \mathrm{~Hz}$ stimulation in a clinically-relevant manner, as previously described (Keller et al., 2018). Each subject received $15 \mathrm{~min}$ of $10 \mathrm{~Hz}$ direct electrical stimulation in a bipolar fashion (biphasic pulses at $100 \mu \mathrm{s} /$ phase). Each stimulation train was $5 \mathrm{~s}$ (50 pulses/train) followed by $10 \mathrm{~s}$ rest $(15 \mathrm{~s}$ duty cycle), resulting in 60 total trains and 3000 total pulses applied (Bakker et al., 2015) (Fig. 1B). The stimulation current used was set at $100 \%$ of the motor threshold in patients with motor cortex coverage (Mehta and Klein, 2010; Keller et al., 2014b). Otherwise, 1-10 mA was chosen depending on patient tolerance. The stimulation parameters were chosen to closely mimic commonly used rTMS treatment paradigms (McClintock et al., 2018). Stimulation sites were chosen in the frontal, temporal and parietal cortices as specified in Table 1. The mean stimulation current across all subjects was $6.1 \mathrm{~mA}$ (SD: 3.0).

\section{Experimental design and statistical analyses}

Temporal dynamics of the stimulation response. To examine cortical responses during the repetitive stimulation protocol both within the stim- ulation train (intra-train) and after the train (post-train), we epoched the ECoG signal surrounding the $5 \mathrm{~s}$ stimulation train $(3 \mathrm{~s}$ before the first pulse and $8 \mathrm{~s}$ after the last pulse). The epoch was subsequently standardized using $Z$-scores against the pre-train baseline period ( -600 to -100 $\mathrm{ms}$ before the start of the first pulse). The stimulation response was defined as the mean response during the period of stimulation (first pulse to last pulse). The offset response was defined as the mean response from 10 $\mathrm{ms}$ (to dissociate the offset response from the stimulation response) to $1000 \mathrm{~ms}$ after the last pulse. To quantify the dynamics of the stimulation response over time, we used repeated-measures ANOVA. First, as evoked oscillations were prominent during the stimulation, we quantified the slow fluctuations in the broadband signal by applying a fourth-order Butterworth $1 \mathrm{~Hz}$ low-pass filter. Second, we divided the stimulation period into $500 \mathrm{~ms}$ bins. We reasoned if cortical excitability was changed during stimulation, then the means of individual bins should be significantly different. These bins represent the within-subject variable. Third, we created a variable representing the stimulation train number. Finally, we fitted a repeated measures model, where the broadband signal as stratified by the time bins is the response and the stimulation train number is the predictor variable (Time Bins $\sim$ Stimulation Train). The $F$ statistic and associated $p$ value were obtained for each coefficient (Time Bins and Time Bins $\times$ Stimulation Train). The $F$ statistic for the Time Bins coefficient indicates whether there was a significant effect of time during the stimulation on the broadband signal whereas the $F$ statistic for the Time Bins-Stimulation Train interaction indicates whether there was progressive modulation of the broadband signal across the 

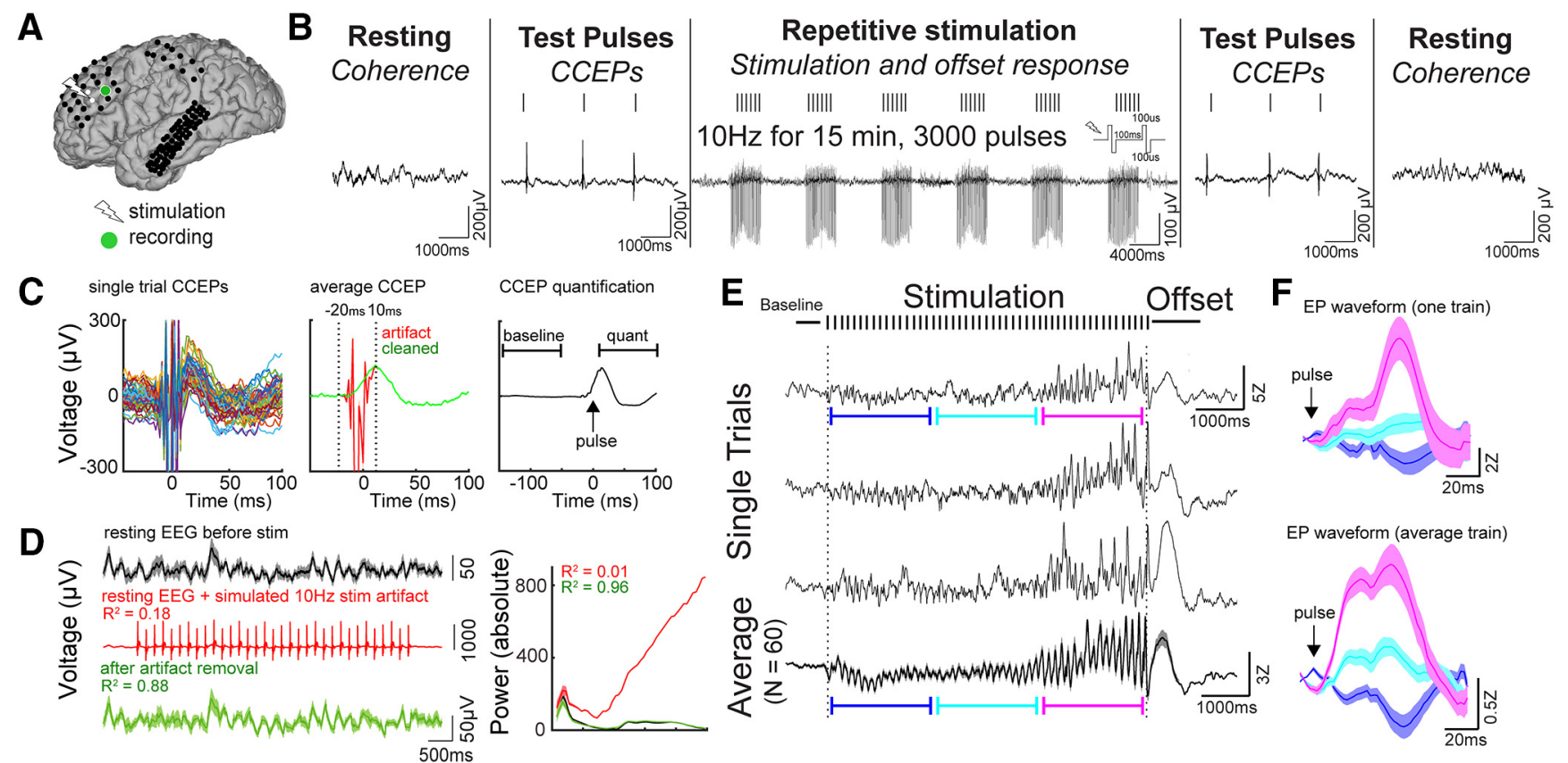

Figure 1. Experimental design and artifact removal. $\boldsymbol{A}$, Intracranial stimulation and recording (Subject 4). Coregistered preoperative MRI and postoperative CT allowed the visualization of subdural electrodes. B, Schematic of the stimulation paradigm. Periods of rest ("Resting") and single-pulse (CEPs ("Test pulses") were applied before and after repetitive stimulation. Focal repetitive stimulation consisted of $15 \mathrm{~min}$ of $5 \mathrm{~s} 10 \mathrm{~Hz}$ trains with an intertrain interval of $6-10 \mathrm{~s}$. Pulses were bipolar with $100 \mu \mathrm{s} / \mathrm{phase}$. ECoG was recorded during each phase of the stimulation paradigm. C, Removal of electrical stimulation artifact. Left, Single-trial CCEP demonstrates the stimulation artifact follows a consistent waveform. Middle, The stimulation artifact identified between - 20 and $10 \mathrm{~ms}$ (shown in red) was removed using our artifact rejection pipeline (see Materials and Methods). The green trace represents the CCEP signal after artifact removal. Right, To quantify CCEPs, we performed Z-standardization of the signal using baseline of -150 to $-50 \mathrm{~ms}$, and subsequently averaged the signal between 10 and $100 \mathrm{~ms}$ across all trials. $\boldsymbol{D}$, Power-frequency analysis using simulated ECOG data to test the effectiveness of the artifact removal process. A $10 \mathrm{~Hz}$ stimulation artifact was added to the resting ECOG data, and the artifacts were subsequently removed using our artifact rejection pipeline (see Materials and Methods). The artifact-spiked data had poor correlation with the original data, whereas the artifact-removed data had good fidelity with the original data. Right, Power spectrum is showed for the original data, the artifact-spiked data, and the cleaned data. The power spectrum of the artifact-spike data are poorly correlated with that of the original data, whereas the spectrum of the artifact-removed data resembled that of the original data. $\boldsymbol{E}$, Single-trial and average ECoG during and directly following the stimulation train recorded from the black electrode labeled in $A$. Note (1) the increase in evoked potential amplitude later in the stimulation train, and (2) the offset response: an evoked potential shortly after the $10 \mathrm{~Hz} \mathrm{stimulation} \mathrm{train.}$ $\boldsymbol{F}$, Evoked potentials after single electrical pulses at different time periods during stimulation. Blue, First third of the evoked potentials; teal, second third of the evoked potentials; magenta, last third of the evoked potentials. Top, Waveforms of evoked potentials using one stimulation train. Bottom, Waveforms of evoked potentials using an average of all stimulation trains. Error bars show \pm 1 standard error of the mean (SEM).

stimulation trains. A channel was considered stimulation responsive if either coefficient was significant using a $p$ value of 0.05 after FDR correction for multiple channels comparison.

Spectral decomposition of intra-stimulation dynamics. We evaluated the time-frequency dynamics during stimulation using Hanning tapers (100 $\mathrm{ms}$ interval, $-1 \mathrm{~s}$ pre-train to $+2 \mathrm{~s}$ post-train). We identified characteristic changes in spectral power of the stimulation train in three frequency ranges: $1-8 \mathrm{~Hz}$ (low-frequency power), $8-40 \mathrm{~Hz}$ (mid-frequency power), and $70-170 \mathrm{~Hz}$ (high-gamma power). These frequency bins were chosen after visual inspection of the time-frequency response because of observed differences in these bins (Fig. 2A). To quantify slow changes in spectral power (Nir et al., 2008; Keller et al., 2013), we first applied a bandpass filter (Butterworth, two-pass) with filter order 4 for lower frequency bands $(1-8 \mathrm{~Hz}$ and $8-40 \mathrm{~Hz})$ or 8 for higher frequency bands $(70-170 \mathrm{~Hz}$; Crowther et al., 2019). Next, we took the absolute value of the filtered signal's Hilbert transform to obtain the analytic signal [often referred to as band-limited power (BLP); Foster et al., 2015]. Finally, we applied a fourth-order Butterworth $1 \mathrm{~Hz}$ low-pass filter to obtain the slow component of the BLP (Nir et al., 2007; Keller et al., 2013) to compare the different BLP signals. Each data point was $Z$-transformed relative to the pre-train baseline period $(-600$ to $-100 \mathrm{~ms}$ before the start of the first pulse) for normalization across patients.

Prestimulation/poststimulation CCEP mapping (effective connectivity). To examine causal changes in brain excitability at baseline and after stimulation, we performed corticocortical evoked potential (CCEP) mapping (Keller et al., 2014a). CCEPs have been used to predict the onset of ictal events (David et al., 2008), examine the functional brain infrastructure (Keller et al., 2011, 2014b; David et al., 2013; Entz et al., 2014), and causally examine the frontoparietal (Matsumoto et al., 2012), hippocampal (Kubota et al., 2013; Mégevand et al., 2017), visual (Keller et al., 2017), and language (Koubeissi et al., 2012) networks. Before and immediately after repetitive stimulation, we applied bipolar electrical stimulation (biphasic pulses at $100 \mu \mathrm{s} /$ phase) with a $1 \mathrm{~s}$ inter-stimulation interval (ISI). This ISI was chosen to allow voltage deflections to return to baseline after $\sim 500 \mathrm{~ms}$ and to allow for sufficient trials to be collected within the expected time constraints to establish a stable prestimulation CCEP baseline (Keller et al., 2018). A uniform random jitter $( \pm 200 \mathrm{~ms}$ ) was included in the ISI to avoid potential entrainment effects that could change baseline cortical excitability (Keller et al., 2018). Stimulation current was chosen to match the current used during repetitive stimulation. $191 \pm 20$ (mean \pm SE) single pulses were applied to assess the baseline CCEP, whereas $662 \pm 80$ pulses were applied to assess poststimulation CCEP. The number of CCEPs was chosen to maximize signal-to-noise within the amount of experimental time allotted. The number of pre and post-CCEPs measured for each subject are outlined in Table 2. CCEPs from each channel were first epoched from -1000 to $1000 \mathrm{~ms}$. The epoch was subsequently standardized using $Z$-scores against the pre-CCEP baseline period ( -150 to $-50 \mathrm{~ms}$; Fig. $1 C$ ) by the following formula: $(x-\mu) / \mathrm{SD}$ where $x$ is a data sample, $\mu$ is the mean of the baseline period, and SD is the SD of the baseline period. The amplitude of the CCEPs was determined by averaging the standardized signal $10-100 \mathrm{~ms}$ after the stimulation pulse and averaging across all trials (Fig. $1 C$ ). To evaluate whether CCEPs evoked at baseline were statistically different from zero, we used cluster-based nonparametric testing as previously described (Maris and Oostenveld, 2007). Briefly, we calculated one-sample $t$ statistics at every time point from 10 to $100 \mathrm{~ms}$ to form clusters of significant 

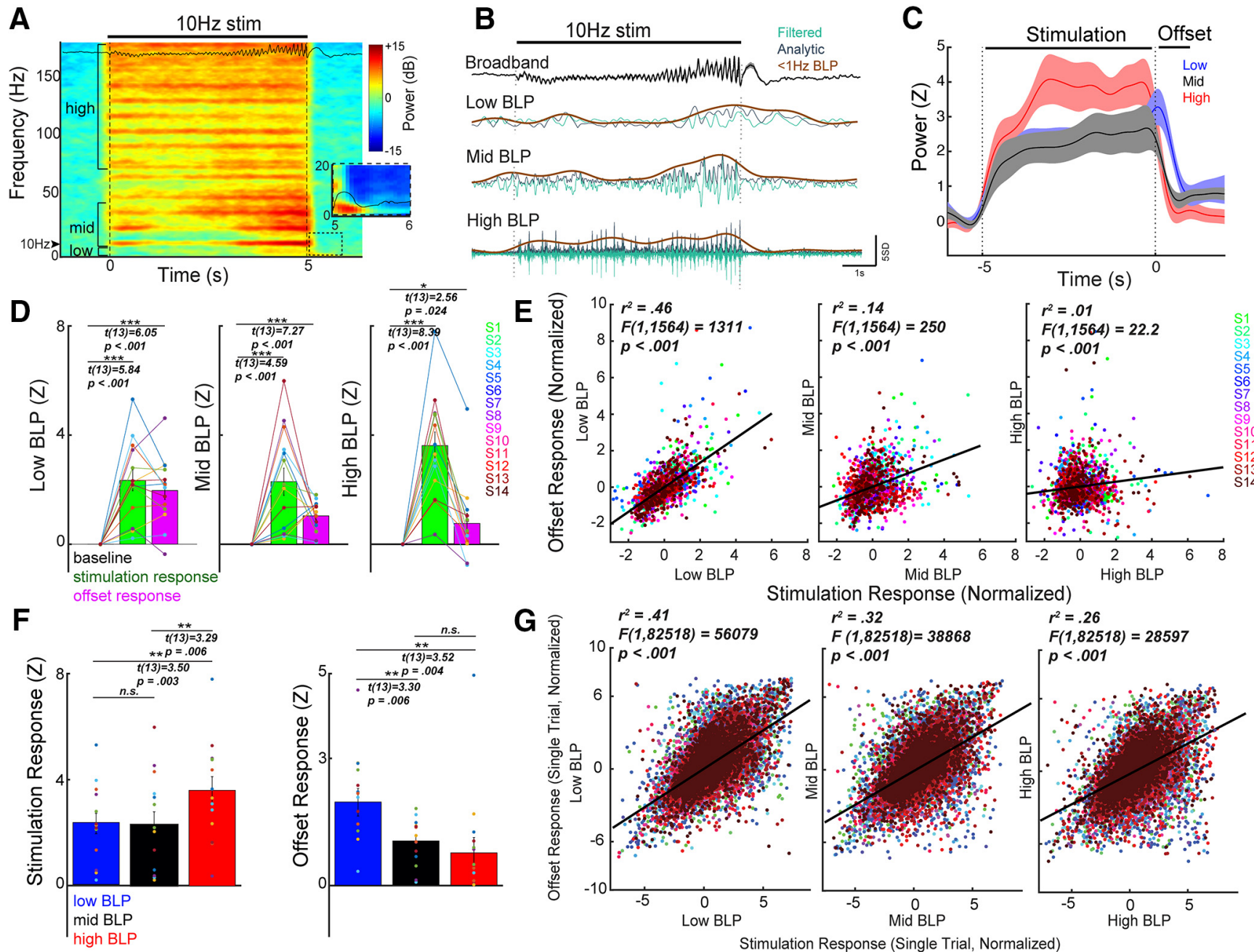

Figure 2. Repetitive stimulation elicits a multiphasic neural response. $\boldsymbol{A}$, Time frequency spectrogram during $10 \mathrm{~Hz}$ stimulation. ECoG data shown in $\boldsymbol{A}$ and $\boldsymbol{B}$ are recorded from the electrode in Figure $1 A$. The stimulation period is characterized by changes in $8-40 \mathrm{~Hz}$ power, corresponding to the changes in evoked potentials observed in the raw broadband signal, as well as an increase in high gamma ( $>70 \mathrm{~Hz}$ ) activity. Immediately after the stimulation train, an evoked response lasting $\sim 1000 \mathrm{~ms}$ occurs, and is primarily driven by low-frequency power ( $<8 \mathrm{~Hz}$; inset). $\boldsymbol{B}$, Raw broadband signal (black) was transformed to BLP to capture temporal dynamics of power changes during stimulation. $\boldsymbol{C}$, Group dynamics of stimulationinduced response. Trials and channel data were averaged per subject, and the subject-averaged trace for each BLP is shown. Mid-BLP ( $8-40 \mathrm{~Hz}$ ) and $70-170 \mathrm{~Hz}$ power (HGP) remain elevated during stimulation and decrease quickly afterward, whereas low BLP (1-8 Hz) increases during stimulation and peaks in the offset period. $\boldsymbol{D}$, Group-level response during different phases of the stimulation train. Across subjects, the mean BLP during stimulation was significantly elevated. In the offset period, low-BLP, mid-BLP and HGP were elevated. $E$, Channel-level relationship between the stimulation response and the offset response for each BLP (black line). Each color represents data from a single subject. Note the strongest correlation between the stimulation response and the offset response was observed with low BLP. $F$, Comparison of BLP in the stimulation and the offset period. During stimulation, the mean HGP was significantly higher than low-BLP and mid-BLP. In the offset period, low-BLP was significantly higher than the mid-BLP and HGP. Each dot represents the channelaveraged response for a single subject. $\mathbf{G}$, The stimulation response is highly correlated with the offset response on a single-trial level. The linear regression line (black) was calculated using the aggregate of all data points. In all three frequency bands, the stimulation response was highly correlated with the offset response on a single-trial basis. Error bars show \pm 1 SEM. ns, not significant, ${ }^{*} p<0.05,{ }^{* *} p<0.01,{ }^{* * *} p<0.001$.

time points based on temporal adjacency at an alpha level of 0.05 (Maris and Oostenveld, 2007). The cluster-level statistics were obtained by taking the sum of the $t$ statistic within each cluster. To generate the null distribution, we calculated the cluster $t$ statistic for randomly shuffled ECoG signals based on 1000 simulations. The cluster $t$ statistic was compared with this null distribution and the CCEP was considered significant using a $p$ value of 0.05 after FDR correction for multiple channels comparison. To determine whether poststimulation CCEPs were significantly different from baseline CCEPs (i.e., to assess presence of poststimulation connectivity changes), we first matched the number of poststimulation CCEP trials with baseline CCEP trials. For example, if 200 baseline CCEP trials were present, then the first 200 poststimulation CCEP trials were used for statistical testing. From 10-100 ms after the single pulse, two-sample $t$ statistic was obtained at every time point and significant clusters were formed based on temporal adjacency at an alpha level of 0.05 . The sum of the $t$ statistic was obtained for each cluster. The null distribution for the cluster $t$ statistic was produced by randomly shuffling trials between baseline CCEPs and poststimulation CCEPs for 1000 iterations and computing the cluster $t$ statistics. The poststimulation CCEPs were considered significantly different from the baseline CCEPs using a cluster $p$ value of 0.05 corrected for multiple channels comparison. The percentage of channels found to have significantly different poststimulation CCEPs for each subject are outlined in Table 2.

Prestimulation/poststimulation coherence analysis (functional connectivity). To estimate functional connectivity through oscillatory synchrony of two brain regions, we computed coherence between all possible electrode pairs using FieldTrip (ft_connectivityanalysis; Maris et al., 2007). Coherence provides a measure of the phase difference between the paired signals and has been previously used to estimate functional connectivity (Solomon et al., 2018; Yazdan-Shahmorad et al., 2018). To calculate coherence, we divided the pre and poststimulation resting pe- 
Table 2. Stimulation setting, recording parameters, and stimulation outcomes for each participant

\begin{tabular}{|c|c|c|c|c|c|c|c|c|c|}
\hline ID & $\begin{array}{l}\text { Stimulation } \\
\text { frequency, } \mathrm{Hz}\end{array}$ & $\begin{array}{l}\text { Stimulation } \\
\text { current, } \mathrm{mA}\end{array}$ & $\begin{array}{l}\text { Channel } \\
\text { No. }{ }^{a}\end{array}$ & $\begin{array}{l}\text { CCEP No., } \\
\text { prestimulation }\end{array}$ & $\begin{array}{l}\text { CCEP No., } \\
\text { poststimulation }\end{array}$ & $\begin{array}{l}\text { Channels that } \\
\text { are stimulation } \\
\text { responsive, } \%^{b}\end{array}$ & $\begin{array}{l}\text { Channel with } \\
\text { significant response } \\
\text { modulation, } \%^{b}\end{array}$ & $\begin{array}{l}\text { Channel with } \\
\text { pre-/post-CCEP } \\
\text { change, } \%^{b}\end{array}$ & $\begin{array}{l}\text { Channel with } \\
\text { pre-/post-theta } \\
\text { coherence change, \%c }\end{array}$ \\
\hline S1 & 10 & 6 & 159 & 149 & 790 & 1.9 & 0.6 & 0 & 0 \\
\hline S2 & 10 & 1.35 & 121 & 149 & 1265 & 18.2 & 7.4 & 0.8 & 0 \\
\hline S3 & 10 & 1 & 166 & 144 & 330 & 0.6 & 0 & 0 & 0 \\
\hline S4 & 10 & 8 & 104 & 194 & 794 & 42.3 & 19.2 & 7.6 & 7.7 \\
\hline S5 & 10 & 7 & 176 & 231 & 868 & 12.5 & 1.1 & 5.1 & 0 \\
\hline S6 & 10 & 10 & 57 & 149 & 448 & 63.2 & 28.1 & 50.9 & 0 \\
\hline S7 & 10 & 10 & 62 & 149 & 448 & 53.2 & 27.4 & 16.1 & 3.2 \\
\hline S8 & 10 & 5 & 26 & 149 & 448 & 26.9 & 0 & 15.4 & 0 \\
\hline$\$ 9$ & 10 & 5 & 64 & 149 & 743 & 21.9 & 1.6 & 0 & 0 \\
\hline S10 & 10 & 10 & 79 & 149 & 449 & 31.7 & 6.3 & 3.8 & 0 \\
\hline S11 & 10 & 8 & 54 & 149 & 297 & 77.8 & 20.4 & 18.5 & 0 \\
\hline S12 & 10 & 6 & 99 & 199 & 400 & 19.2 & 8.1 & 3.0 & 0 \\
\hline S13 & 10 & 4 & 197 & 358 & 997 & 18.8 & 7.6 & 0.5 & 1.5 \\
\hline S14 & 10 & 4 & 202 & 358 & 1000 & 1.5 & 0.5 & 1.0 & 7.9 \\
\hline
\end{tabular}

${ }^{a}$ Bipolar montage was used for depth electrodes (stimulation channels and noise channels excluded).

${ }^{b}$ The variations in these percentages across patients are dependent on the stimulation current. The correlation coefficient are as follows: $R_{c}$

$: 0.70(p=0.006) ; R$

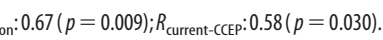
The theta frequency band used is $4-8 \mathrm{~Hz}$ with 29 of 1566 total channels showing change. This analysis was performed for other frequency bands including $8-12,12-25,25-50$, and $70-100 \mathrm{~Hz}$. The numbers of channels showing change in these frequency bands were $30,1,3$, and 8 of 1566 channels, respectively.

riods ranging from 5 to 10 min into $1 \mathrm{~s}$ epochs. We used a multitaper method with $2 \mathrm{~Hz}$ spectral smoothing to compute the spectral estimate of each epoch (Scheeringa et al., 2011; Yazdan-Shahmorad et al., 2018). Coherence was calculated as the normalized cross-spectral density between the two signals. Theta coherence was obtained by averaging across frequency range of $4-8 \mathrm{~Hz}$ and was used in the primary analysis. Theta coherence has been previously used in both nonhuman primates and human studies to measure functional connectivity (Solomon et al., 2018; Yazdan-Shahmorad et al., 2018). Alpha $(8-12 \mathrm{~Hz})$, beta $(12-25 \mathrm{~Hz})$, gamma $(25-50 \mathrm{~Hz})$, and high gamma $(70-100 \mathrm{~Hz})$ frequency bands were also used for comparison analyses. To determine whether prestimulation coherence for a particular pair of channels was significant, we generated a null distribution. To do this, we divided the resting time series into 20 bins, randomly shuffled the bins, and subsequently computed theta coherence. This bin number was chosen to be large enough to maintain the temporal structure of the ECoG time series but small enough to allow multiple iterations of data shuffling. This procedure was repeated for 1000 iterations. Coherence for a particular pair of channels was considered significant using a $p$ value of 0.01 after FDR correction for multiple channels comparison. To test for coherence differences prestimulation and poststimulation, we used nonparametric testing as described previously (Maris et al., 2007). Briefly, we calculated the Z-transformed coherence statistic and generated the null-distribution of the difference in coherence by randomly shuffling among prestimulation and poststimulation trials. This procedure was repeated 1000 times. Coherence was considered significantly different between two conditions using a $p$ value of 0.05 after FDR correction for multiple channels comparison. The percentage of channels found to have significantly different poststimulation theta coherence (to the stimulation site) for each subject are outlined in Table 2.

Prediction of poststimulation connectivity change. To determine whether features during the stimulation period predicted connectivity changes, we used logistical regression. For this analysis, we pooled all channels into a single dataset and categorized the channels by two outcomes: if there was significant pre-/post-CCEP change or significant pre-/post-theta coherence change. For a particular channel, the features derived from the stimulation period included (1) the stimulation response (the mean voltage during the stimulation period), (2) the presence of a significant stimulation response, and (3) the presence of modulation in the stimulation response after repeated stimulation trains. To evaluate the proportion of stimulation data required for good model performance, we created six subsets of the data. Using 1\% (9 s), 10\% (1.5 $\mathrm{min}$ ), 20\% (3 $\mathrm{min}$ ), 60\% (9 $\mathrm{min}$ ), 85\% (12.75 min), and 100\% (15 min) of the stimulation trains, we derived the three features from the stimulation period. For each subset, we performed logistic regression with ten- fold cross validation. Receiver operating characteristic (ROC) curves were constructed and we quantified area under the curve (AUC) to evaluate model performance. To allow for comparison among models using different subset of data, we used bootstrap sampling (1000 permutations) to estimate the mean and $95 \%$ confidence interval of the model AUC.

\section{Results}

We performed direct electrical stimulation using implanted electrodes while simultaneously recording electrical activity from the cortical surface (ECoG). Individual patient characteristics and stimulation sites are listed in Table 1. Electrode locations for each patient is visualized in Figure 5. As shown in Figure 1, $A$ and $B$, our stimulation paradigm included resting periods to evaluate functional connectivity, test pulses to evaluate effective connectivity, and repetitive stimulation. This paradigm provided us the unique opportunity to assess neural activity before, during, and after stimulation.

\section{Repetitive stimulation elicits a characteristic neural response}

To study plasticity induction, we characterized neural activity occurring during repetitive electrical stimulation. We constructed a robust artifact rejection procedure using principles previously validated for CCEPs (Fig. 1C; see Materials and Methods; Crowther et al., 2019). To assess the validity of this procedure on stimulation artifacts during a stimulation train, we used a previously described simulation approach by adding stimulation artifacts to resting ECoG data (Amengual et al., 2017; Fig. $1 D)$. Poor correlation was observed between the artifact-spiked data and the original data $\left(R^{2}=0.18\right)$, whereas good fidelity was observed between the artifact-removed data and the original data (Fig. $1 D ; R^{2}=0.88$ ). After artifact rejection, the power spectrum of the cleaned ECoG data resembled that of the original resting data (Fig. $1 D ; R^{2}=0.96$ ). We demonstrated a consistent neural response to stimulation following artifact rejection, on both a single trial and average level (Fig. 2E). This stimulation response consisted of an increasing evoked potential amplitude (Fig. $1 F$ ) and a slow shift in voltage during the stimulation train. Immediately poststimulation train, we observed an evoked response lasting $\sim 1 \mathrm{~s}$ (offset response). We further characterized this signal by computing the time-frequency power spectrum (Fig. 2A). High 
gamma power (HGP; 70-170 Hz) was elevated throughout the stimulation train, while $8-40 \mathrm{~Hz}$ power varied, which reflected the changing amplitude of the evoked potential after each pulse. The offset response showed primarily an increase in lowfrequency power $(1-8 \mathrm{~Hz})$. To explore the temporal dynamics of these power changes during stimulation on coarse timescales, we computed slow changes in BLP (Fig. 2B). Averaged over all channels and subjects, HGP and mid-BLP $(8-40 \mathrm{~Hz})$ rapidly increased during stimulation and decreased quickly afterward, whereas low BLP $(1-8 \mathrm{~Hz})$ gradually increased during stimulation and peaked during the offset period (Fig. 2C). To quantify these changes, we averaged the response during stimulation $(-5$ to $0 \mathrm{~s}$ ) and during the offset period $(0.01-1 \mathrm{~s})$. We found that stimulation elicited significant mean response in all BLP during stimulation (Fig. 2D; see for statistical details, Table 3). Similar results were obtained when conventional frequency bands were used (Table 3). We then determined whether a particular BLP was higher than the other during stimulation and found HGP $(70-170 \mathrm{~Hz})$ to demonstrate a stronger response compared with low BLP (Fig. 2F, left; Table 3) or mid-BLP. The offset period showed a significant increase in low BLP (Fig. 2D, left; Table 3), mid-BLP (Fig. 2D, middle), and HGP (Fig. 2D, right). Conventional frequency band analyses revealed the offset response showed significant power increases in the delta, theta, and alpha bands, but not beta and gamma bands (Table 3). Further, low BLP was significantly higher than both mid-BLP (Fig. $2 F$, right; Table 3) and HGP in the offset period.

We next asked whether the offset response could be used as a proxy for the response during stimulation, as determining the response during stimulation in other modalities such as rTMS can be challenging because of the multitude of stimulation-related artifacts (Wu et al., 2018). Thus, we evaluated the relationships between the stimulation response and the offset response using linear regression. The strongest relationship was observed between the stimulation and the offset responses in low BLP (Fig. 2E, left; $R^{2}=0.46$; Table 3 ), followed by mid-BLP (Fig. $2 E$, middle; $R^{2}=0.14$ ). The weakest relationship was found in HGP (Fig. $2 E$, right; $R^{2}=0.01$ ). On a single-trial level, the relationship between stimulation response and offset response was highest for low BLP (Fig. 2G, left; $R^{2}=0.41$; Table 3), followed by mid-BLP (Fig. $2 G$, middle; $R^{2}=0.32$ ) and HGP (Fig. $2 G$, right; $R^{2}=0.26$ ). Finally, we repeated the above analyses using raw voltage (broadband response) as these data would be easily accessible from a clinical perspective. Repetitive stimulation elicited a significant broadband response during stimulation (Table 3 ) and in the offset period. Correlation was observed between the stimulation and offset response on both a channel $\left(R^{2}=0.20\right.$; Table $3)$ and single-trial level $\left(R^{2}=0.50\right)$.

In summary, repetitive stimulation elicits a measurable response both during and immediately after the stimulation train. The stimulation response is characterized by predominantly an increase in HGP whereas the offset response is driven primarily by low-frequency power. The offset response is best correlated with the stimulation response using low-frequency power; however, robust correlations were observed across all bands on a single-trial level.

\section{Stimulation response is predicted by effective and functional connectivity}

We next asked how the stimulation response relates to inter-area connectivity before stimulation. We quantified connectivity using single-pulse CCEPs (a measure of network response to elec- trical stimulation) and resting theta coherence (a measure of related spontaneous neural activity). Qualitatively, channels with strong prestimulation CCEPs also exhibited strong broadband stimulation responses (Fig. $3 A, B$ ) in one exemplar subject (Subject 4 ). Further, the relationship among prestimulation theta coherence (to the stimulation site), CCEP, and the stimulation response is spatially shown (Fig. $3 C-F)$. To relate prestimulation connectivity to the stimulation response, we plotted the stimulation response stratified by the strength of connectivity. We found that regions with stronger prestimulation CCEPs also demonstrated stronger broadband stimulation responses (Fig. 3G, left; Table 3). Likewise, regions with higher prestimulation theta coherence also showed stronger broadband stimulation response (Fig. $3 G$, right). We repeated this analysis for the HGP stimulation response (Fig. $3 H$ ) and found that HGP stimulation response was significantly higher in regions with higher prestimulation CCEPs (Fig. $3 \mathrm{H}$, left; Table 3 ) and theta coherence (Fig. $3 H$, right). To avoid using an arbitrary threshold and to generalize this finding across subjects, we next defined whether or not a particular channel showed significant baseline CCEPs or theta coherence to the stimulation site (see Materials and Methods). We found that across subjects the broadband stimulation response was consistently stronger in regions with significant prestimulation CCEPs (Fig. 3I, left; Table 3) and theta coherence to the stimulation site (Fig. 3I, right). In a similar manner, the HGP during stimulation was higher in regions with significant prestimulation CCEPs (Fig. 3J, left; Table 3) and theta coherence to the stimulation site (Fig. 3J, right). To test whether these results were dependent on the coherence band used, we computed coherence using conventional frequency bands. Channels with significant band coherence in all frequency bands showed stronger broadband stimulation response (Table 3; all $p<0.05$ ). Higher HGP stimulation response was observed in channels with significant prestimulation alpha, beta, and gamma coherence (Table 3; all $p<0.01$ ), but not high-gamma coherence. Further, the increase in HGP or broadband stimulation response in regions with significant CCEP or theta coherence was not different when stratified by sites of stimulation (motor, frontal, or temporal; Table 3). In summary, prestimulation network connectivity defined by CCEPs and coherence across frequency bands predicts the neural response during stimulation.

\section{Stimulation response is modulated after repeated stimulation in regions highly connected to the stimulation sites}

As shown in Figure $1 B$, our stimulation paradigm included 60 repeated applications of $10 \mathrm{~Hz}$ stimulation trains separated by resting periods. This allowed us to track changes in neural activity during each stimulation train. We observed that the broadband signal during stimulation starts changing around train 10 and peaks in amplitude around train 40 (Fig. 4B). Compared with early stimulation trains, later stimulation trains elicited progressively more negative response at the beginning of the train, and more positive response toward the end of the train (Fig. 4B). To isolate the gradual change in brain excitability, we obtained the slow fluctuations $(<1 \mathrm{~Hz})$ in the broadband signal, which effectively removes the faster evoked potentials (Fig. 4C). Subsequently, we quantified the stimulation dynamics using a repeated-measures model incorporating time bins and stimulation train as variables (see Materials and Methods; Fig. 4D). A significant interaction between time during stimulation and the stimulation train number $\left(\right.$ Ftime $\left.\times \operatorname{train}_{(7399)}=3.49, p=0.001\right)$ indicated that there was modulation of the stimulation response over repeated trains. Across all channels, 19.7\% of channels (308/ 
Table 3. Statistical estimate and $p$ value referenced in figures and results

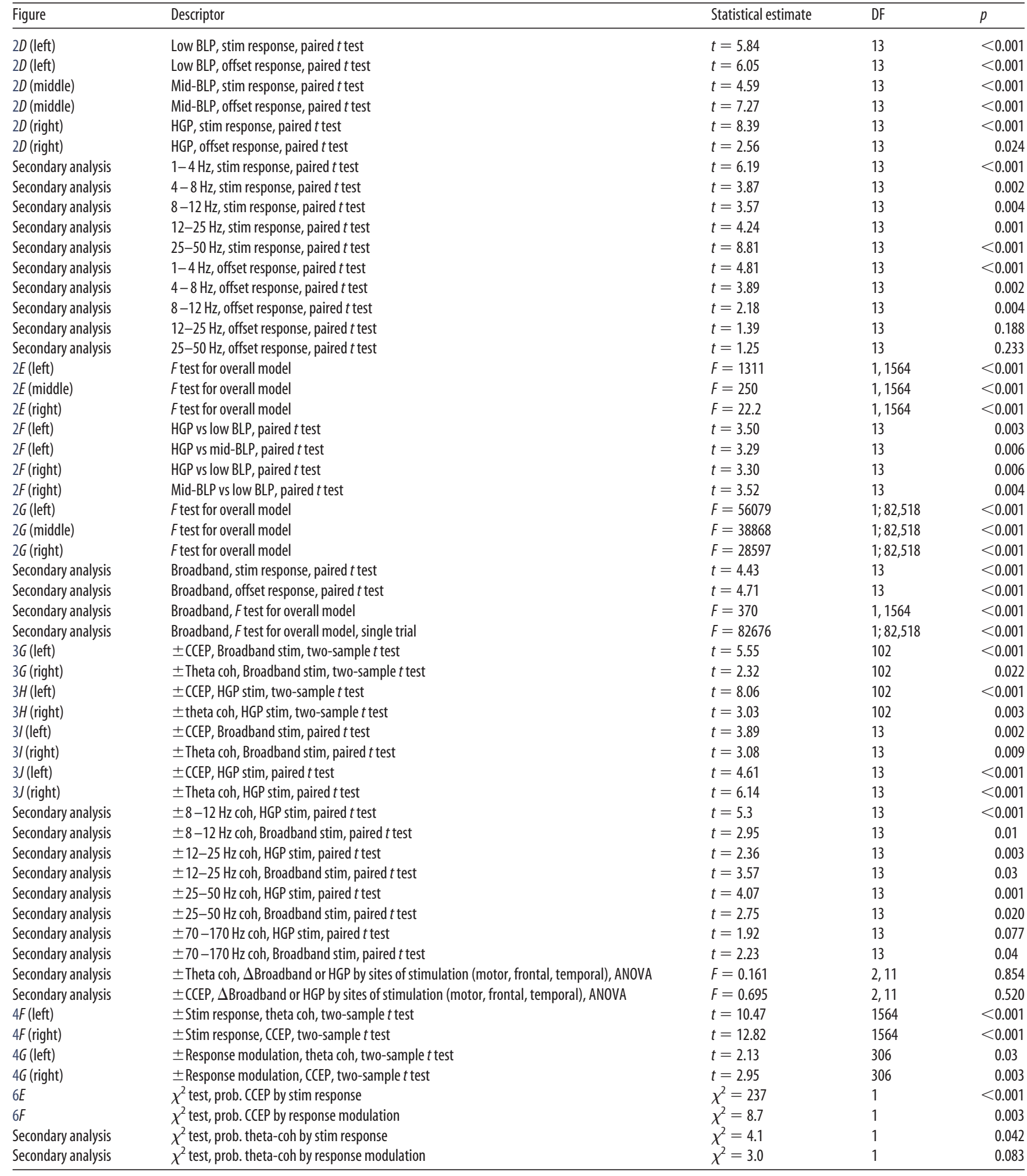

coh, coherence; prob, probability

1566) were stimulation responsive, which is defined as significance of either the time or time $\times$ train coefficients in the repeated-measures model (Fig. $4 E$ ). Within these stimulation responsive channels, 34.4\% (106/308) showed progressive modulation of the stimulation response. Spatially, these stimulation responsive channels were primarily local to the stimulation site (Fig. 5). On a single-subject level, the stimulation current ampli- tude correlated strongly both with the number of stimulation responsive channels $\left(R^{2}=0.49, p=0.006\right.$; Table 2$)$ and the proportion of channels exhibiting response modulation $\left(R^{2}=\right.$ $0.45, p=0.009$ ). Pooling together all channels, stimulation responsive channels exhibited higher prestimulation theta coherence (Fig. 4F, left; Table 3 ) and CCEP (Fig. 4F, right) compared with nonresponsive channels. Furthermore, limiting the analysis 
A

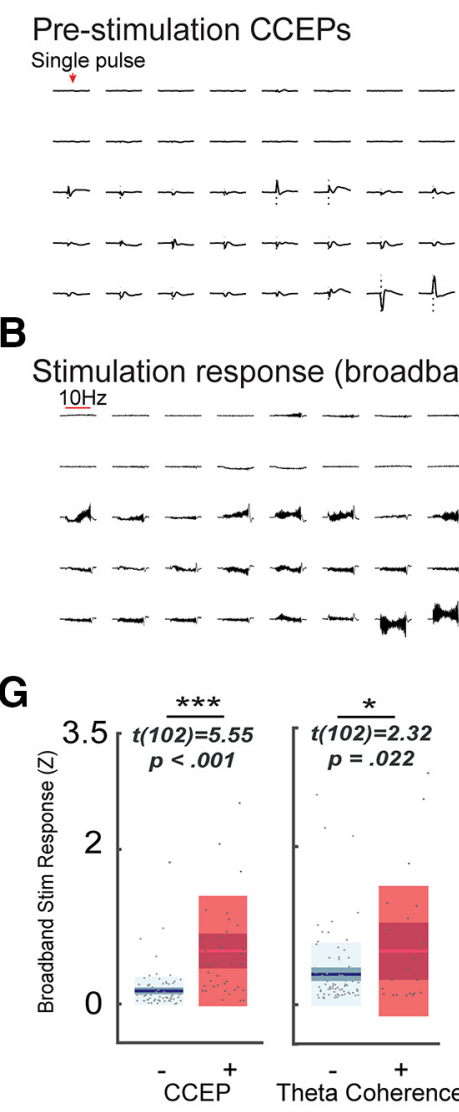

C

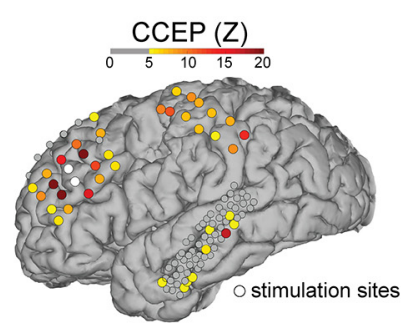

E

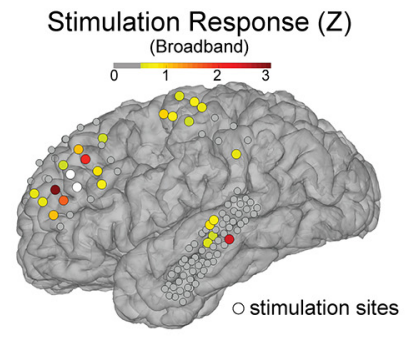

D

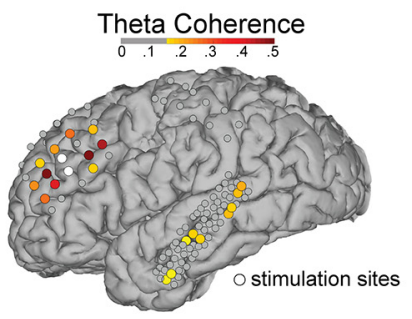

$\mathbf{F}$
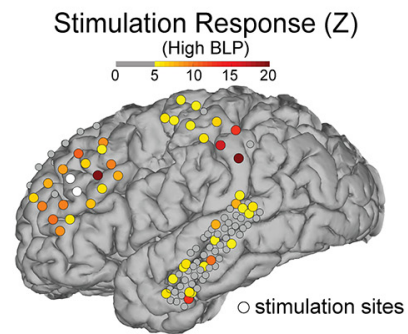
$\overrightarrow{3 s}^{\vec{N}}$

Figure 3. The stimulation response is predicted by functional and effective connectivity. $\boldsymbol{A}, \boldsymbol{B}$, Exemplar broadband signal across several channels for Subject 4. $A$, Single-pulse CCEPs recorded before stimulation and $(\boldsymbol{B})$ the corresponding neural response to the stimulation train. Qualitatively electrodes with strong CCEPs generally also elicited strong response during the stimulation train. $\mathbf{C}-\boldsymbol{F}$, Single subject (Subject 4) spatial distribution of CCEP, theta coherence and the stimulation response (broadband and HGP). $\boldsymbol{C}$, Strong (CEPs were elicited near the stimulation sites and at select parietal and temporal regions. $\boldsymbol{D}$, Theta coherence to the stimulation site was highest in the prefrontal cortex. $\boldsymbol{E}$, The mean voltage during stimulation (the broadband stimulation response) was high in certain regions across prefrontal, parietal, and temporal cortices. $\boldsymbol{F}$, Similar pattern of response was observed for HGP during stimulation. $\mathbf{G}$, Increased mean broadband response was observed during stimulation in channels with higher prestimulation CCEP (left; + refers to $\geq 5 Z$ and - refers to $<5 Z$ ) and theta coherence to the stimulation site (right; + refers to coherence of $\geq 0.15$ and - refers to $<0.15$ ). $\boldsymbol{H}$, Box plots demonstrating increased mean HGP during stimulation in channels with higher prestimulation CCEP (left) and theta coherence to the stimulation site (right). $I, J$, Channels with significant prestimulation CCEP or theta coherence were averaged per subject, and the mean stimulation response is shown for each subject. I, Higher broadband stimulation response is observed in channels with significant prestimulation CCEP (left) and theta coherence to the stimulation site (right) across subjects. J, Higher HGP stimulation response is observed in channels with significant prestimulation CCEP (left) and theta coherence to the stimulation site (right) across subjects. Error bars show \pm 1 SEM. ${ }^{*} p<0.05,{ }^{* *} p<0.01,{ }^{* * *} p<0.001$. Box plots show the mean value (innermost line), the $95 \% \mathrm{Cl}$ (dark band), and the SD (light band).

to only the stimulation responsive channels $(N=308)$, those channels which demonstrated modulation of the stimulation response over time had higher prestimulation theta coherence (Fig. $4 G$, left; Table 3 ) and CCEP (Fig. 4G, right) compared with channels that were stimulation responsive but did not undergo modulation of activity across stimulation trains. In summary, stimulation-responsive regions were more strongly connected to the stimulation site than nonresponsive regions. In a subset of regions that were strongly connected to the stimulation site, repeated stimulation trains progressively modulated neural activity over time.

\section{The stimulation period predicts changes in poststimulation connectivity}

Finally, we asked whether features pertaining to the stimulation period can be used to predict poststimulation connectivity changes. We hypothesized that stimulation responsive regions and regions that exhibit modulation of the stimulation response would be more likely to show poststimulation connectivity changes. For instance, single $10 \mathrm{~Hz}$ stimulation trains elicited a strong stimulation response, and repeated trains elicited progressive modulation of the response (Fig. 6A,B). We also observed a significant increase in the CCEP after the entire repetitive stimulation protocol (Fig. 6C; nonparametric cluster $t$ test, cluster $T=$ $631.15, p<0.001)$. Of note, the direction of amplitude change observed in the pre-/post-CCEP (here, stronger poststimulation CCEP) mirrored that of the direction of modulation (stronger) of the broadband stimulation response (Fig. $6 B, C$ ). In total, a small proportion $(5.1 \%, 80 / 1566)$ of all channels showed a significant pre-/post-CCEP change (Fig. 6D). Likewise, a small proportion $(1.9 \%, 29 / 1566)$ of all channels demonstrated significant change in pre/post-theta coherence. We found that stimulation responsive channels were more likely to undergo pre-/post-CCEP change (Fig. $6 E ; 22.0 \%$ vs $0.90 \%$; Table 3 ). Similarly, of only the stimulation responsive channels, channels where stimulation response was progressively modulated were more likely to show pre-/post-CCEP change (Fig. 6F; 32.0 vs 17.0\%). Additionally, we performed this analysis looking at poststimulation changes in 

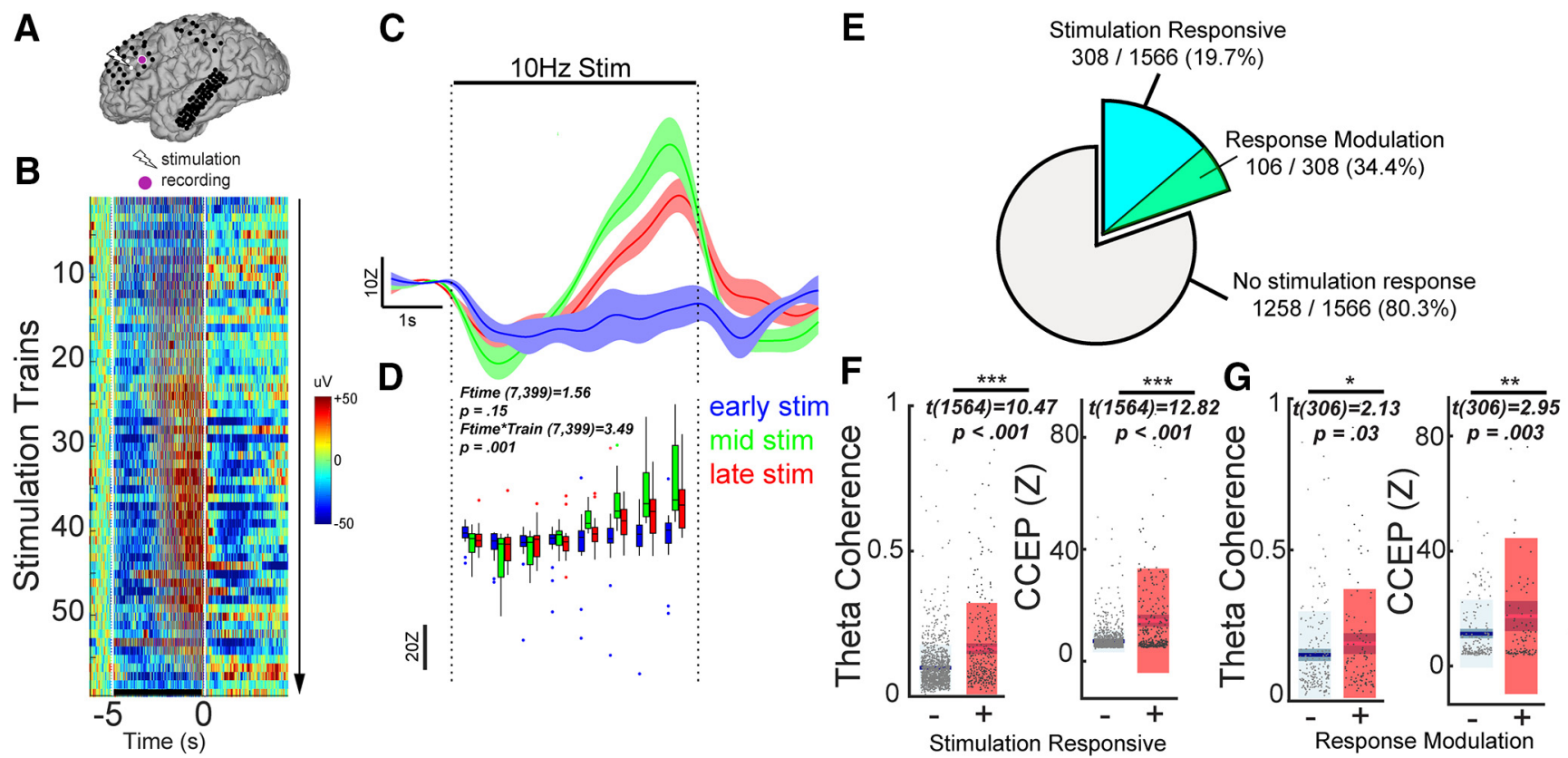

Figure 4. Progressive modulation of the stimulation response occurs in regions highly connected to the stimulation site. $A-D$, Example of neural changes across stimulation trains in one subject (Subject 4). $\boldsymbol{A}$, Location of stimulation and the recording electrode. $\boldsymbol{B}$, Heatmap representation of the epoched broadband signal to increasing number of stimulation trains. Horizontal line represents time period of stimulation train. Color in the image represents the broadband signal during and after stimulation train. $C$, Time series of the smoothed broadband signal during stimulation as stratified by early (blue), middle (green), and late (red) trains in the stimulation protocol. D, Quantification of the stimulation dynamics in the exemplar channel. Repeated-measures ANOVA demonstrated a significant interaction between time during stimulation and the stimulation train number. $\boldsymbol{E}$, Among aggregate of all channels across 14 patients, 308/1566 (19.7\%) of channels were stimulation-responsive. Among the 308 stimulation-responsive channels, 106 (34.4\%) showed a modulation in stimulation response (for location of these channels, see Fig. 5). $\boldsymbol{F}$, Box plots stratifying prestimulation CCEP and theta coherence by stimulation responsive channels. Stimulation responsive channels demonstrated higher theta coherence (left) and CCEP amplitude (right). $G$, Box plots stratifying prestimulation CCEP and theta coherence by channels that did or did not undergo response modulation. Channels with stimulation response modulated by progressive trains demonstrated higher theta coherence (left; two-samplettest, $p=0.03$ ) and CCEP amplitude (right). Error bars show \pm 1 SEM. ${ }^{*} p<0.05,{ }^{* *} p<0.01,{ }^{* * *} p<0.001$. Boxplots show the mean value (innermost line), the 95\% Cl (dark band), and the SD (light band).
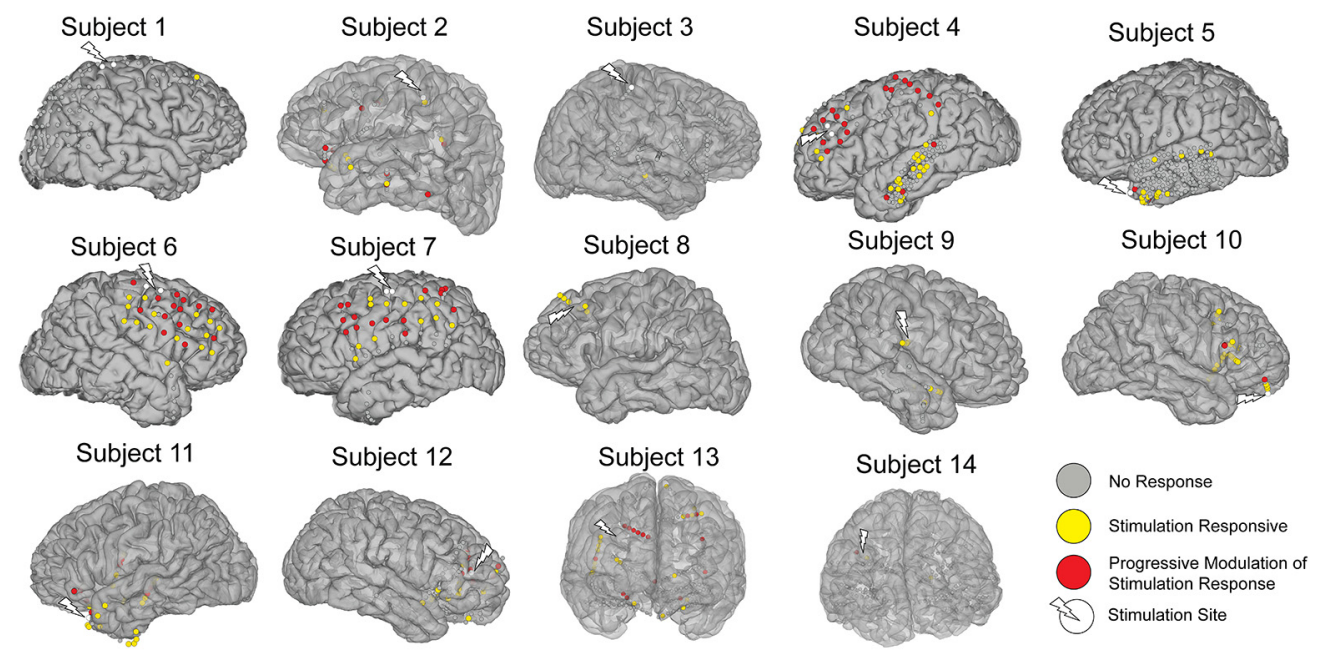

Figure 5. Location of electrodes exhibiting significant stimulation responses and modulation of the stimulation response. In each subject, significant stimulation responses were elicited in a subset of the regions recorded (yellow and red electrodes). Of all the stimulation-responsive electrodes, some underwent significant change in the stimulation response over repeated application of the stimulation train (red electrodes). Regions demonstrating response modulation are primarily local to the stimulation sites, although a small proportion of these electrodes are found on the opposite hemisphere or distant cortex.

theta coherence. The presence of a significant stimulation response was also associated with poststimulation change in theta coherence $(3.3 \%$ vs $1.5 \%$; Table 3$)$. The presence of response modulation was not associated with pre-/post-theta coherence change ( 5.7 vs $2.0 \%)$. We next used logistic regression to assess whether poststimulation connectivity changes can be predicted from features during stimulation. The features used were (1) the presence of a significant stimulation response without response modulation (binary variable), (2) the presence of response modulation (binary), and (3) amplitude of the broadband stimulation response (continuous). Channels were categorized as local ( $\leq 4$ $\mathrm{cm}$ from the stimulation site) and remote ( $\leq 4 \mathrm{~cm}$ from the stimulation site). Subsets of the stimulation duration were used to determine the minimal number of stimulation trains required for 

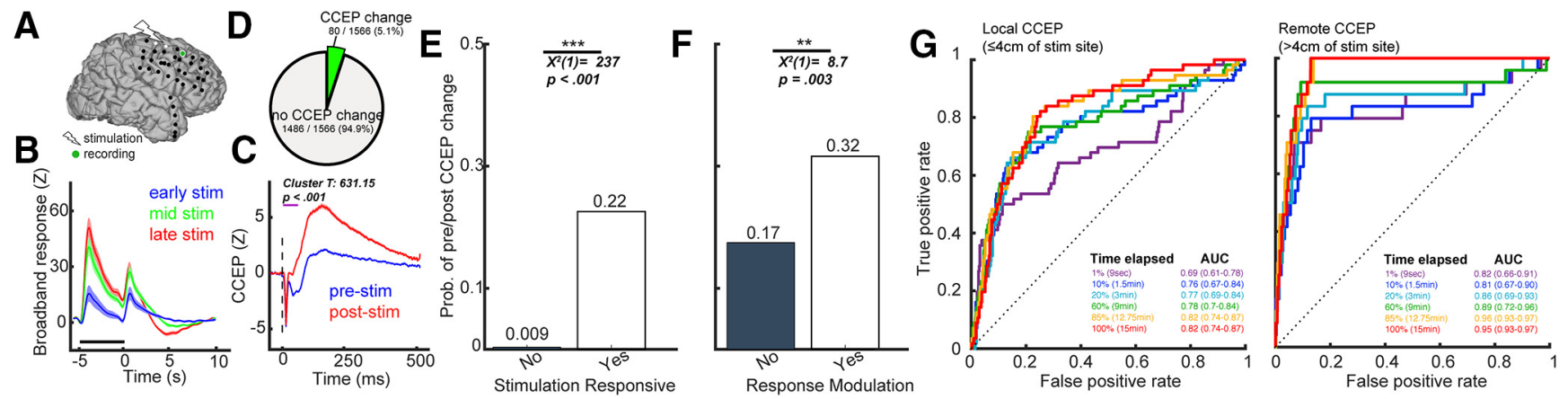

Figure 6. The stimulation period predicts connectivity changes following the entire stimulation protocol. $\boldsymbol{A}-\boldsymbol{C}$, Exemplar channel recording from Subject $6 . \boldsymbol{A}$, Location of stimulation and the recording electrode. $\boldsymbol{B}$, Time series of the smoothed broadband signal during stimulation as stratified by early (blue), middle (green), and late (red) trains in the stimulation protocol. $\boldsymbol{C}$, The corresponding pre-/post- single-pulse CCEP. Note the similar direction of change in CCEP and in the stimulation response. D, Among aggregate of all channels across 14 patients, 80/1566 (5.1\%) of channels showed significant pre-/post-CCEP change. $\boldsymbol{E}$, The probability of CCEP change in regions with and without a significant stimulation response. Stimulation responsive channels had higher probability of showing pre-/post-CCEP change. $\boldsymbol{F}$, The probability of CCEP change in regions with and without response modulation by stimulation trains. Among only the stimulation-responsive channels, those that showed response modulation had a higher probability of showing pre-/post-CCEP change. G, Receiver Operating Characteristic (ROC) curves using features from the stimulation period to predict pre-/post-CCEP change. The features used were the presence of significant stimulation response without response modulation, the presence of response modulation by stimulation trains and the mean amplitude of the broadband signal during stimulation. Left, Prediction of CCEP change in channels closer than $4 \mathrm{~cm}$ of the stimulation site. Right, Prediction of CCEP change in channels further than $4 \mathrm{~cm}$ of the stimulation site. Greater performance was achieved in predicting remote CCEP changes. Error bars show \pm 1 SEM. ${ }^{* *} p<.01,{ }^{* * *} p<0.001$.

stable model performance. Using 1, 10, 20, 60, 85 , and $100 \%$ of the stimulation data, poststimulation changes in local CCEP were predicted with AUC 0.69 (95\% confidence interval: 0.61-0.78), $0.76(0.67-0.84), 0.77(0.69-0.84), 0.78(0.70-0.84), 0.82(0.74-$ $0.87), 0.82(0.74-0.87)$, respectively (Fig. $6 G$, left). The model AUCs were not significantly different from each as the confidence intervals overlap. For remote CCEP changes using 1, 10, 20, 60, 85 , and $100 \%$ of the stimulation data, the AUC values were 0.82 $(0.66-0.91), 0.81(0.67-0.90), 0.86(0.69-0.93), 0.89(0.72-$ $0.96), 0.96(0.93-0.97), 0.95(0.93-0.97)$, respectively. Aside from the 1 and $10 \%$ data subset, the AUC for all other subsets were not significantly different. We were not able to meaningfully predict poststimulation changes in theta coherence $[\mathrm{AUC}=0.47$ (0.37-0.61) using $100 \%$ of data and both local and remote channels]. In summary, changes in CCEP and theta coherence occurred in a small proportion of total channels. Features from the stimulation period predicted CCEP changes after the stimulation protocol with high discriminability. Using subsets of the stimulation trains, we showed that model performance can reach stability with only a small proportion of the total data.

\section{Discussion}

In this study we investigated the neural dynamics during and after a series of repeated stimulation trains. Across several stimulation sites, we observed a consistent increase in HGP activity during the stimulation train and a slower post-train evoked response that strongly correlated with activity changes during the train. We showed that in areas highly connected to the stimulation site, as indexed by two measures of connectivity, the stimulation response was stronger and exhibited progressive modulation with repeated trains. Finally, the stimulation period predicted poststimulation connectivity changes with high discriminability. Importantly, we demonstrated that using a subset of the stimulation protocol was sufficient for good model performance.

Mounting evidence suggests that the induction period is characterized by stimulation-driven cycles of excitation and inhibition. In this study, we expand on evidence from animal studies during the induction period and offered insight into how a clinically-relevant stimulation pattern influences brain dynamics. First, we observed an increase in HGP during stimulation, especially in regions functionally connected to the stimulation site. As HGP has been shown to correlate with spiking activity (Nir et al., 2007; Manning et al., 2009; Ray and Maunsell, 2011), this work suggests that $10 \mathrm{~Hz}$ stimulation elicits increases in neuronal activity during stimulation. These findings are similar to recent work in rat hippocampal slices, which reported a slow voltage drift during high-frequency stimulation that corresponded with the change in EPSP amplitude (Wójtowicz and Mozrzymas, 2014). Second, low-frequency power also increased during $10 \mathrm{~Hz}$ stimulation. Although this is in contrast with a recent study that reported stimulation-driven decreases (Rao et al., 2018), this may be attributed to the difference in frequency of stimulation (10 vs $100 \mathrm{~Hz}$ ). Finally, after each stimulation train, we observed an evoked potential lasting for $\sim 1 \mathrm{~s}$, which we termed the offset response. Consistent with a prior study, this offset response is primarily driven by low-frequency power that is not dependent on the stimulation frequency. (Solomon et al., 2018). These slow waves likely represent GABA-ergic inhibitory periods (Ulbert et al., 2004), which have been observed during spike and wave discharges (McCormick and Contreras, 2001; Alarcón et al., 2012) as well as single-pulse evoked potentials in animals (Chen et al., 2014) and humans (Alarcón et al., 2012; Keller et al., 2014a). Together, these findings suggest that in regions functionally connected to the stimulation site, stimulation trains increased spiking activity, which were followed by an inhibitory rebound period.

Previously, we demonstrated that repetitive brain stimulation results in excitability changes that outlast the stimulation period itself (Keller et al., 2018). However, what happens during stimulation remains unexplored. Our current study revealed that neural response to repetitive stimulation is highly dynamic both within a single stimulation train and across multiple stimulation trains. Within a single train, evoked potentials became progressively stronger, manifesting as increased power across multiple frequency bands. Across trains, slow fluctuations in broadband power were progressively modulated, suggesting a short-term change in the underlying excitability of the brain in response to stimulation. These results offer evidence that repetitive stimulation may rapidly reorganize neural connectivity, even within minutes. Furthermore regions that demonstrated dynamic changes during stimulation were more likely to show sustained 
changes after stimulation. Similar findings have been reported in nonhuman primates and in the human motor cortex. In nonhuman primates, repetitive optogenetic stimulation strengthened functional connectivity between motor and somatosensory cortices within minutes (Yazdan-Shahmorad et al., 2018). In the same study, stimulation-evoked activity predicted poststimulation changes in connectivity. Similarly, in humans, motor evoked potential (MEP) facilitation during anodal transcranial direct current stimulation predicted lasting MEP changes poststimulation (Bergmann et al., 2009). Our results along with these prior studies support the notion that plasticity following brain stimulation follows Hebbian mechanisms, and that depending on the stimulation site, different regions are more susceptible to stimulation-induced changes.

Of note, although we were able to predict poststimulation CCEP changes with high discriminability, our model performance was not as robust in the prediction of poststimulation theta coherence changes. This could be due to the order of poststimulation testing (resting data for coherence was collected after CCEP testing and therefore was further in time from the stimulation protocol) or the method of connectivity measurement (evoked for CCEPs, rest for coherence analysis). Although it was beyond the scope of this investigation, comparing theta coherence and CCEP in approximating network connectivity would be of substantial interest.

The clear neurophysiological effects we observed during stimulation offer intriguing clinical utility. Efforts to optimize treatment by updating stimulation parameters in real-time have recently sparked an interest in closed-loop brain stimulation (Bergmann et al., 2016; Bergmann, 2018; Ghasemi et al., 2018). Two findings from this study highlight the potential for real-time implementation. First, we found that the strength of stimulation response was correlated strongly with the strength of the offset response, both on a channel and single-trial level. This is an important finding given removal of stimulation artifacts in other modalities (i.e., EEG during rTMS; Wu et al., 2018) is often difficult. Our findings suggest that the offset response may be used as a proxy for the stimulation response during clinical monitoring. Second, we found that only a few minutes of stimulation data were required to achieve stable model performance in predicting poststimulation effects. To date, we lack a method to rapidly optimize stimulation patterns for an individual, as pre-/posttesting after the entire stimulation protocol will be cumbersome if multiple parameters are tested. Yet based on our results, if the stimulation response (or offset response) can be monitored and pre-processed in real-time or near real-time, then effects of multiple stimulation paradigms can be tested rapidly. If this work can be replicated by noninvasive modalities, such as TMS, then one could envision a "stimulation localizer" day before stimulation treatment. During this day, prestimulation characteristics would first be used to help localize the stimulation site (and network) of interest (Keller et al., 2018). This would be followed by multiple stimulation trains of short duration ( $2 \mathrm{~min}$ ) with various parameters (frequency, pattern, intensity) to select the stimulation paradigm that would maximize poststimulation effects.

Several aspects of this study limit its generalizability. First, because seizures can alter both local and global brain excitability and connectivity (Pereira et al., 2010; Bettus et al., 2011; Pittau et al., 2012), our results may not be entirely representative of responses in a healthy brain. Although direct recording provides unsurpassed spatiotemporal resolution in humans, epilepsy patients differ in their underlying etiologies and electrode implantation patterns. Second, behavioral effects of stimulation were not measured in this study and this warrants further investigation with mood self-reporting (Woźniak-Kwaśniewska et al., 2014). Third, given hospital time constraints (typically $\sim 1 \mathrm{~h}$ per patient), we were unable to modify the stimulation parameters that may be critical in inducing plasticity: stimulation site, frequency, and intensity. Fourth, we observed prominent harmonics during stimulation, which are due to evoked potentials in response to the electrical pulses. Although one could attempt to model and remove harmonics, we believe that they represent progressive modulation of the evoked potential during stimulation and are a direct result of electrical stimulation. As such, we recognize the power increase during stimulation can arise from two sources: (1) the evoked potentials and (2) an underlying increase in neuronal excitability, which could not be separated in the present study.

Future work includes (1) computational modeling of evoked responses and their spectral profile, allowing us to disentangle stimulation responses from pulse-evoked neuronal activity and that of the underlying neuronal activity, providing a way to study entrainment effects during repetitive stimulation; (2) a thorough examination of how parameters of a single stimulation trainincluding train frequency, number of pulses, and intensity-affect stimulation and offset responses; (3) an evaluation of how both induction and poststimulation effects are modulated when repeated trains (3000 pulses each) are applied at different stimulation frequencies $(10$ and $100 \mathrm{~Hz}$, for example), stimulation intensities (50 and 100\% MT), and stimulation sites; (4) an evaluation of not only how these stimulation parameters affect mood, similar to Rao et al. (2018), but also for several stimulation frequencies and targets; (5) replication of these experiments using microelectrode recording in nonhuman primates to evaluate mechanistic changes at a scale unattainable even in ECoG; and (6) adapting these experiments to patients with neuropsychiatric disorders using noninvasive neuromodulation (TMS) paired with scalp EEG, to determine whether these signals can be feasibly measured and monitored for clinical translation. Knowledge gained from these planned experiments will greatly enhance our understanding of how stimulation modulates human brain activity and behavior, helping propel us to the next generation of personalized neuromodulation therapies.

Here, we characterized the neural activity in the time period surrounding a train of electrical stimulation and its dependency on existing functional networks, thus providing valuable insight as to how the brain changes during stimulation. Furthermore, we demonstrated the utility of this information, by showing that neural activity during stimulation serves to predict stimulationinduced changes in network connectivity. Together, our work provides key insights into the development of closed-loop neuromodulatory devices.

\section{References}

Alarcón G, Martinez J, Kerai SV, Lacruz ME, Quiroga RQ, Selway RP, Richardson MP, García Seoane JJ, Valentín A (2012) In vivo neuronal firing patterns during human epileptiform discharges replicated by electrical stimulation. Clin Neurophysiol 123:1736-1744.

Amengual JL, Vernet M, Adam C, Valero-Cabré A (2017) Local entrainment of oscillatory activity induced by direct brain stimulation in humans. Sci Rep 7:41908.

Bakker N, Shahab S, Giacobbe P, Blumberger DM, Daskalakis ZJ, Kennedy SH, Downar J (2015) rTMS of the dorsomedial prefrontal cortex for major depression: safety, tolerability, effectiveness, and outcome predictors for $10 \mathrm{~Hz}$ versus intermittent theta-burst stimulation. Brain Stimul $8: 208-215$

Bergmann TO (2018) Brain state-dependent brain stimulation. Front Psychol 9:2108.

Bergmann TO, Groppa S, Seeger M, Mölle M, Marshall L, Siebner HR (2009) 
Acute changes in motor cortical excitability during slow oscillatory and constant anodal transcranial direct current stimulation. J Neurophysiol 102:2303-2311.

Bergmann TO, Karabanov A, Hartwigsen G, Thielscher A, Siebner HR (2016) Combining non-invasive transcranial brain stimulation with neuroimaging and electrophysiology: current approaches and future perspectives. Neuroimage 140:4-19.

Bettus G, Ranjeva JP, Wendling F, Bénar CG, Confort-Gouny S, Régis J, Chauvel P, Cozzone PJ, Lemieux L, Bartolomei F, Guye M (2011) Interictal functional connectivity of human epileptic networks assessed by intracerebral EEG and BOLD signal fluctuations. PLoS One 6:e20071.

Bliss TV, Collingridge GL (1993) A synaptic model of memory: long-term potentiation in the hippocampus. Nature 361:31-39.

Bliss TV, Lomo T (1973) Long-lasting potentiation of synaptic transmission in the dentate area of the anaesthetized rabbit following stimulation of the perforant path. J Physiol 232:331-356.

Chen CH, Fremont R, Arteaga-Bracho EE, Khodakhah K (2014) Short latency cerebellar modulation of the basal ganglia. Nat Neurosci 17:1767-1775.

Crowther LJ, Brunner P, Kapeller C, Guger C, Kamada K, Bunch ME, Frawley BK, Lynch TM, Ritaccio AL, Schalk G (2019) A quantitative method for evaluating cortical responses to electrical stimulation. J Neurosci Methods 311:67-75.

Dale AM, Fischl B, Sereno MI (1999) Cortical surface-based analysis: I. segmentation and surface reconstruction. Neuroimage 9:179-194.

David O, Woźniak A, Minotti L, Kahane P (2008) Preictal short-term plasticity induced by intracerebral $1 \mathrm{~Hz}$ stimulation. Neuroimage 39:16331646.

David O, Job AS, De Palma L, Hoffmann D, Minotti L, Kahane P (2013) Probabilistic functional tractography of the human cortex. Neuroimage 80:307-317.

Duncan JS, Papademetris X, Yang J, Jackowski M, Zeng X, Staib LH (2004) Geometric strategies for neuroanatomic analysis from MRI. Neuroimage 23:S34-S45.

Dykstra AR, Chan AM, Quinn BT, Zepeda R, Keller CJ, Cormier J, Madsen JR, Eskandar EN, Cash SS (2012) Individualized localization and cortical surface-based registration of intracranial electrodes. Neuroimage 59: 3563-3570

Entz L, Tóth E, Keller CJ, Bickel S, Groppe DM, Fabó D, Kozák LR, Erőss L, Ulbert I, Mehta AD (2014) Evoked effective connectivity of the human neocortex. Hum Brain Mapp 35:5736-5753.

Fischl B, van der Kouwe A, Destrieux C, Halgren E, Ségonne F, Salat DH, Busa E, Seidman LJ, Goldstein J, Kennedy D, Caviness V, Makris N, Rosen B, Dale AM (2004) Automatically parcellating the human cerebral cortex. Cereb Cortex 14:11-22.

Foster BL, Rangarajan V, Shirer WR, Parvizi J (2015) Intrinsic and taskdependent coupling of neuronal population activity in human parietal cortex. Neuron 86:578-590.

Ghasemi P, Sahraee T, Mohammadi A (2018) Closed- and open-loop deep brain stimulation: methods, challenges, current and future aspects. J Biomed Phys Eng 8:209-216.

Groppe DM, Bickel S, Dykstra AR, Wang X, Mégevand P, Mercier MR, Lado FA, Mehta AD, Honey CJ (2017) iELVis: an open source MATLAB toolbox for localizing and visualizing human intracranial electrode data. J Neurosci Methods 281:40-48.

Hamidi M, Slagter HA, Tononi G, Postle BR (2010) Brain responses evoked by high-frequency repetitive transcranial magnetic stimulation: an eventrelated potential study. Brain Stimul 3:2-14.

Huang YY, Colino A, Selig DK, Malenka RC (1992) The influence of prior synaptic activity on the induction of long-term potentiation. Science 255 : $730-733$.

Keller CJ, Bickel S, Entz L, Ulbert I, Milham MP, Kelly C, Mehta AD (2011) Intrinsic functional architecture predicts electrically evoked responses in the human brain. Proc Natl Acad Sci U S A 108:10308-10313.

Keller CJ, Bickel S, Honey CJ, Groppe DM, Entz L, Craddock RC, Lado FA, Kelly C, Milham M, Mehta AD (2013) Neurophysiological investigation of spontaneous correlated and anticorrelated fluctuations of the BOLD signal. J Neurosci 33:6333-6342.

Keller CJ, Honey CJ, Mégevand P, Entz L, Ulbert I, Mehta AD (2014a) Mapping human brain networks with cortico-cortical evoked potentials. Philos Trans R Soc Lond B Biol Sci 369:20130528.

Keller CJ, Honey CJ, Entz L, Bickel S, Groppe DM, Toth E, Ulbert I, Lado FA,
Mehta AD (2014b) Corticocortical evoked potentials reveal projectors and integrators in human brain networks. J Neurosci 34:9152-9163.

Keller CJ, Davidesco I, Megevand P, Lado FA, Malach R, Mehta AD (2017) Tuning face perception with electrical stimulation of the fusiform gyrus. Hum Brain Mapp 38:2830-2842.

Keller CJ, Huang Y, Herrero JL, Fini ME, Du V, Lado FA, Honey CJ, Mehta $\mathrm{AD}$ (2018) Induction and quantification of excitability changes in human cortical networks. J Neurosci 38:5384-5398.

Koubeissi MZ, Lesser RP, Sinai A, Gaillard WD, Franaszczuk PJ, Crone NE (2012) Connectivity between perisylvian and bilateral basal temporal cortices. Cereb Cortex 22:918-925.

Kozyrev V, Eysel UT, Jancke D (2014) Voltage-sensitive dye imaging of transcranial magnetic stimulation-induced intracortical dynamics. Proc Natl Acad Sci U S A 111:13553-13558.

Kubota Y, Enatsu R, Gonzalez-Martinez J, Bulacio J, Mosher J, Burgess RC, Nair DR (2013) In vivo human hippocampal cingulate connectivity: a corticocortical evoked potentials (CCEPs) study. Clin Neurophysiol 124:1547-1556.

Liu J, Fukunaga K, Yamamoto H, Nishi K, Miyamoto E (1999) Differential roles of $\mathrm{Ca}^{2+} /$ calmodulin-dependent protein kinase II and mitogenactivated protein kinase activation in hippocampal long-term potentiation. J Neurosci 19:8292-8299.

Manning JR, Jacobs J, Fried I, Kahana MJ (2009) Broadband shifts in local field potential power spectra are correlated with single-neuron spiking in humans. J Neurosci 29:13613-13620.

Maris E, Oostenveld R (2007) Nonparametric statistical testing of EEG- and MEG-data. J Neurosci Methods 164:177-190.

Maris E, Schoffelen JM, Fries P (2007) Nonparametric statistical testing of coherence differences. J Neurosci Methods 163:161-175.

Matsumoto R, Nair DR, Ikeda A, Fumuro T, Lapresto E, Mikuni N, Bingaman W, Miyamoto S, Fukuyama H, Takahashi R, Najm I, Shibasaki H, Lüders HO (2012) Parieto-frontal network in humans studied by corticocortical evoked potential. Hum Brain Mapp 33:2856-2872.

McCormick DA, Contreras D (2001) On the cellular and network bases of epileptic seizures. Annu Rev Physiol 63:815-846.

McClintock SM, Reti IM, Carpenter LL, McDonald WM, Dubin M, Taylor SF Cook IA, O'Reardon J, Husain MM, Wall C, Krystal AD, Sampson SM, Morales O, Nelson BG, Latoussakis V, George MS, Lisanby SF: National Network of Depression Centers rTMS Task Group; American Psychiatric Association Council on Research Task Force on Novel B, Treatments (2018) Consensus recommendations for the clinical application of repetitive transcranial magnetic stimulation (rTMS) in the treatment of depression. J Clin Psychiatry 76:16cs10905.

Mégevand P, Groppe DM, Bickel S, Mercier MR, Goldfinger MS, Keller CJ, Entz L, Mehta AD (2017) The hippocampus and amygdala are integrators of neocortical influence: a corticocortical evoked potential study. Brain Connect 7:648-660.

Mehta AD, Klein G (2010) Clinical utility of functional magnetic resonance imaging for brain mapping in epilepsy surgery. Epilepsy Res 89:126-132.

Miyamoto E (2006) Molecular mechanism of neuronal plasticity: induction and maintenance of long-term potentiation in the hippocampus. J Pharmacol Sci 100:433-442.

Mulkey RM, Malenka RC (1992) Mechanisms underlying induction of homosynaptic long-term depression in area CA1 of the hippocampus. Neuron 9:967-975.

Nir Y, Fisch L, Mukamel R, Gelbard-Sagiv H, Arieli A, Fried I, Malach R (2007) Coupling between neuronal firing rate, gamma LFP, and BOLD fMRI is related to interneuronal correlations. Curr Biol 17:1275-1285.

Nir Y, Mukamel R, Dinstein I, Privman E, Harel M, Fisch L, Gelbard-Sagiv H, Kipervasser S, Andelman F, Neufeld MY, Kramer U, Arieli A, Fried I, Malach R (2008) Interhemispheric correlations of slow spontaneous neuronal fluctuations revealed in human sensory cortex. Nat Neurosci 11:1100-1108.

Oostenveld R, Fries P, Maris E, Schoffelen JM (2011) FieldTrip: open source software for advanced analysis of MEG, EEG, and invasive electrophysiological data. Comput Intell Neurosci 2011:156869.

Pereira FR, Alessio A, Sercheli MS, Pedro T, Bilevicius E, Rondina JM, Ozelo HF, Castellano G, Covolan RJ, Damasceno BP, Cendes F (2010) Asymmetrical hippocampal connectivity in mesial temporal lobe epilepsy: evidence from resting state fMRI. BMC Neurosci 11:66.

Pittau F, Grova C, Moeller F, Dubeau F, Gotman J (2012) Patterns of altered 
functional connectivity in mesial temporal lobe epilepsy. Epilepsia 53:1013-1023.

Rao VR, Sellers KK, Wallace DL, Lee MB, Bijanzadeh M, Sani OG, Yang Y, Shanechi MM, Dawes HE, Chang EF (2018) Direct electrical stimulation of lateral orbitofrontal cortex acutely improves mood in individuals with symptoms of depression. Curr Biol 28:3893-3902.e4.

Ray S, Maunsell JH (2011) Different origins of gamma rhythm and highgamma activity in macaque visual cortex. PLoS Biol 9:e1000610.

Rosanova M, Casarotto S, Pigorini A, Canali P, Casali AG, Massimini M (2012) Combining transcranial magnetic stimulation with electroencephalography to study human cortical excitability and effective connectivity. In: Neuronal network analysis. Neuromethods, Vol 67 (Fellin T, Halassa M, eds) pp 435-457. New York: Humana.

Scheeringa R, Fries P, Petersson KM, Oostenveld R, Grothe I, Norris DG, Hagoort P, Bastiaansen MC (2011) Neuronal dynamics underlying high- and low-frequency EEG oscillations contribute independently to the human BOLD signal. Neuron 69:572-583.

Solomon EA, Kragel JE, Gross R, Lega B, Sperling MR, Worrell G, Sheth SA, Zaghloul KA, Jobst BC, Stein JM, Das S, Gorniak R, Inman CS, Seger S, Rizzuto DS, Kahana MJ (2018) Medial temporal lobe functional connectivity predicts stimulation-induced theta power. Nat Commun 9:4437.

Stolk A, Griffin S, van der Meij R, Dewar C, Saez I, Lin JJ, Piantoni G, Schoffelen JM, Knight RT, Oostenveld R (2018) Integrated analysis of ana- tomical and electrophysiological human intracranial data. Nat Protoc 13:1699-1723.

Ulbert I, Heit G, Madsen J, Karmos G, Halgren E (2004) Laminar analysis of human neocortical interictal spike generation and propagation: current source density and multiunit analysis in vivo. Epilepsia $45: 48-56$.

Veniero D, Maioli C, Miniussi C (2010) Potentiation of short-latency cortical responses by high-frequency repetitive transcranial magnetic stimulation. J Neurophysiol 104:1578-1588.

Wójtowicz T, Mozrzymas JW (2014) Matrix metalloprotease activity shapes the magnitude of EPSPs and spike plasticity within the hippocampal CA3 network. Hippocampus 24:135-153.

Woźniak-Kwaśniewska A, Szekely D, Aussedat P, Bougerol T, David O (2014) Changes of oscillatory brain activity induced by repetitive transcranial magnetic stimulation of the left dorsolateral prefrontal cortex in healthy subjects. Neuroimage 88:91-99.

Wu W, Keller CJ, Rogasch NC, Longwell P, Shpigel E, Rolle CE, Etkin A (2018) ARTIST: a fully automated artifact rejection algorithm for singlepulse TMS-EEG data. Hum Brain Mapp 39:1607-1625.

Yazdan-Shahmorad A, Silversmith DB, Kharazia V, Sabes PN (2018) Targeted cortical reorganization using optogenetics in nonhuman primates. eLife 7:e31034. 